\title{
Ultracold bosons in zig-zag optical lattices
}

\author{
S. Greschner, L. Santos, and T. Vekua \\ Institut für Theoretische Physik, Leibniz Universität Hannover, 30167 Hannover, Germany \\ (Received 23 February 2012; revised manuscript received 22 January 2013; published 6 March 2013)
}

\begin{abstract}
Ultracold bosons in zig-zag optical lattices present a rich physics due to the interplay between frustration induced by lattice geometry, two-body interactions, and a three-body constraint. Unconstrained bosons may develop chiral superfluidity and become a Mott insulator even at vanishingly small interactions. Bosons with a three-body constraint allow for a Haldane-insulator phase in nonpolar gases, as well as pair superfluidity and density-wave phases for attractive interactions. These phases may be created and detected within the current state-of-the-art techniques.
\end{abstract}

DOI: 10.1103/PhysRevA.87.033609

PACS number(s): 67.85.Fg, 64.70.Tg, 67.85.Bc

\section{INTRODUCTION}

Atoms in optical lattices offer extraordinary possibilities for the controlled emulation and analysis of lattice models and quantum magnetism [1,2]. Various lattice geometries are attainable by means of proper laser arrangements, including triangular [3] and Kagome [4] lattices, opening fascinating possibilities for the study of geometric frustration, which may result in flat bands with the constrained mobility and largely enhanced role of interactions [5]. Moreover, the value and sign of intersite hopping may be modified by means of shaking techniques [6,7], allowing for the study of frustrated antiferromagnets with bosonic lattice gases [8].

Interatomic interactions may be controlled basically at will by means of Feshbach resonances [9]. In particular, large on-site repulsion may allow for the suppression of double occupancy in bosonic gases at low fillings (hard-core regime). Interestingly, it has been recently suggested that, due to a Zeno-like effect, large three-body loss rates may result in an effective three-body constraint, in which no more than two atoms may occupy a given lattice site [10]. This constraint opens exciting novel scenarios, including color superfluids in spinor Fermi gases [11] and especially in what concerns stable Bose gases with attractive on-site interactions showing pair superfluidity [10,12-14]. The suppression of three-body occupation has been hinted in recent experiments [15].

Under proper conditions, lattice gases may resemble to a large extent effective spin models, e.g., hard-core bosons may be mapped onto a spin-1/2 XY Heisenberg model [1]. Lattice bosons at unit filling resemble to a large extent spin-1 chains [16] and, in the presence of intersite interactions as is the case of polar gases [17], have been shown to present a gapped Haldane-like phase [18] [dubbed a Haldane-insulator (HI) $[16,19]]$ characterized by a nonlocal string order [20].

In this work we analyze the physics of ultracold bosons in zig-zag optical lattices. We show that the interplay of frustration and interactions lead to a different physics for unconstrained and constrained (with up to two particles per site) bosons. For unconstrained bosons, geometric frustration induces chiral superfluidity and allows for a Mott-insulator phase even at vanishingly small interactions. For constrained bosons, we show that a Haldane-insulator phase becomes possible even for nonpolar gases. Moreover, pair-superfluid $[10,12,14]$ and density-wave phases may occur for attractive on-site interactions. A direct first-order phase transition from
Haldane insulator to pair superfluid is observed and explained. These phases may be realized and detected with existing state-of-the-art techniques.

The structure of the paper is as follows: In Sec. II we introduce the zig-zag lattice model under consideration. Section III is devoted to the unconstrained case, whereas Sec. IV deals with bosons with a two-body constraint. Finally, in Sec. V we summarize our conclusions. Further details on analytical and numerical procedures are discussed in the appendices.

\section{ZIG-ZAG LATTICES}

In the following we consider bosons in zig-zag optical lattices. As shown in Fig. 1, this particular geometry may result from the incoherent superposition of a triangular lattice with elementary cell vectors $\vec{a}_{1}=a \vec{e}_{x}$ and $\vec{a}_{2}=a\left(\frac{1}{2} \vec{e}_{x}+\frac{\sqrt{3}}{2} \vec{e}_{y}\right)$ [formed by three laser beams of wave number $k=4 \pi /(3 a)$ oriented at 120 degrees from each other, as discussed in Ref. [3] and a superlattice with lattice spacing $\sqrt{3} a$ oriented along $y$. For a sufficiently strong superlattice, zig-zag ladders are formed, and the hopping between ladders may be neglected. We will hence concentrate in the following on the physics of bosons in a single zig-zag ladder, which to a large extent is given by the rates $t$ and $t^{\prime}$ characterizing the hopping along the two directions $\vec{a}_{1}$ and $\vec{a}_{2}$ (Fig. 1). As shown in Ref. [8], a periodic lattice shaking may be employed to control the value of $t$ and $t^{\prime}$ independently. Interestingly, their sign may be controlled as well. In the following we consider an inverted sign for both hoppings, which results in an antiferromagnetic coupling between sites [8].

Ordering the sites as indicated in Fig. 1, the physics of the system is given by a Bose-Hubbard Hamiltonian with on-site interactions characterized by the coupling constant $U$, nearestneighbor hopping $t<0$, and next-nearest-neighbor hopping $t^{\prime}<0$ :

$$
\begin{aligned}
H= & \sum_{i}\left[-\frac{t}{2} b_{i}^{\dagger} b_{i+1}-\frac{t^{\prime}}{2} b_{i}^{\dagger} b_{i+2}+\text { H.c. }\right] \\
& +\frac{U}{2} \sum_{i} n_{i}\left(n_{i}-1\right)+U_{3} \sum_{i} n_{i}\left(n_{i}-1\right)\left(n_{i}-2\right),
\end{aligned}
$$

where $b_{i}^{\dagger}, b_{i}$ are the bosonic creation and annihilation operators, respectively, of particles at site $i, n_{i}=b_{i}^{\dagger} b_{i}$, and we 


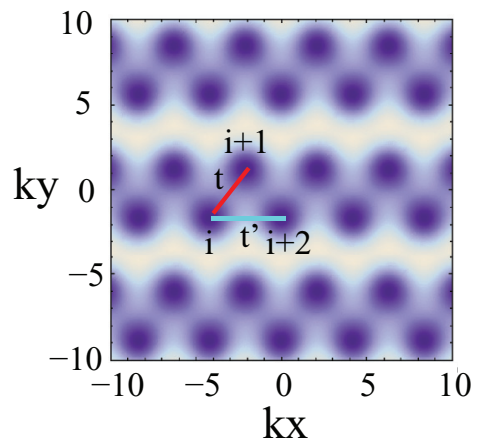

FIG. 1. (Color online) Zig-zag chains formed by an incoherent superposition between a triangular lattice [3] $V_{1}(\vec{r} \equiv(x, y))=$ $V_{10}\left\{\sin ^{2}\left(\vec{b}_{1} \cdot \vec{r} / 2\right)+\sin ^{2}\left(\vec{b}_{2} \cdot \vec{r} / 2\right)+\sin ^{2}\left[\left(\vec{b}_{1}-\vec{b}_{2}\right) \cdot \vec{r} / 2\right]\right\}$, with $k$ being the laser wave number, $\vec{b}_{1}=\sqrt{3} k \vec{e}_{y}$ and $\vec{b}_{2}=\sqrt{3} k\left(\sqrt{3} \vec{e}_{x} / 2-\right.$ $\left.\vec{e}_{y} / 2\right)$, and an additional lattice $V_{2}(\vec{r})=V_{20} \sin ^{2}(\sqrt{3} k y / 4-\pi / 4)$. In the figure, in which $V_{20} / V_{10}=2$, darker regions mean lower potential. The hopping rate between nearest (next-nearest) neighbors is $t>\left(t^{\prime}\right)$.

have added the possibility of three-body interactions, which are characterized by the coupling constant $U_{3}$. We assume below an average unit filling $\bar{n}=1$.

\section{UNCONSTRAINED BOSONS}

We discuss first the ground-state properties of unconstrained bosons $\left(U_{3}=0\right)$. At $U=0$, the Hamiltonian (1) is diagonalized in quasimomentum space $H=\sum_{k} \epsilon(k) b_{k}^{\dagger} b_{k}$, with the dispersion $\epsilon(k)=|t|(\cos k+j \cos 2 k)$, with $j \equiv t^{\prime} / t$. Depending on the frustration $j$ we may distinguish two regimes. If $j<1 / 4$, the dispersion $\epsilon(k)$ presents a single minimum at $k=\pi$, and hence small $U$ will introduce a superfluid (SF) phase, with a quasicondensate at $k=\pi$. If $j>1 / 4, \epsilon(k)$ presents two nonequivalent minima at $k=$ $k_{0} \equiv \pm \arccos [-1 / 4 j]$. As shown below, interactions favor the predominant population of one of these minima, and the system enters a chiral superfluid (CSF) phase with a nonzero local boson current characterized by a finite chirality $\left\langle\kappa_{i}\right\rangle$, with $\kappa_{i}=\frac{i}{2}\left(b_{i}^{\dagger} b_{i+1}-\right.$ H.c. $)$. At $j=1 / 4$, the Lifshitz point, the dispersion becomes quartic at the $k=\pi$ minimum, $\epsilon(k) \sim(k-\pi)^{4}$, the effective mass $m=\left[\partial^{2} \epsilon(k) / \partial k^{2}\right]_{k=\pi}^{-1}=$ $1 / t(1-4 j)$ diverges, and even vanishingly small interactions become relevant.

To study the effect of interactions we combine numerical calculations based on the density matrix renormalization group (DMRG) method [21] (with up to $N=300$ sites keeping per block on average 400 states for gapped phases and 600 states for gapless phases), and bosonization techniques to unveil the low-energy behavior of model (1). For $j<1 / 4$, we employ standard bosonization transformations [22], with an additional oscillating factor $b_{i} \rightarrow(-1)^{i} e^{i \sqrt{\pi} \theta(x)}$, to obtain the low-energy effective theory, which is given by the sine-Gordon model

$$
\mathcal{H}=\frac{v_{s}}{2}\left[\frac{\left(\partial_{x} \phi\right)^{2}}{K}+K\left(\partial_{x} \theta\right)^{2}\right]-\mathcal{M} \cos [2 \pi \bar{n} x-\sqrt{4 \pi} \phi],
$$

where $\theta$ and $\partial_{x} \phi$ describe phase and density fluctuations of bosons, respectively, $\left[\theta(x), \partial_{y} \phi\right]=i \delta(x-y), v_{s}$ is the
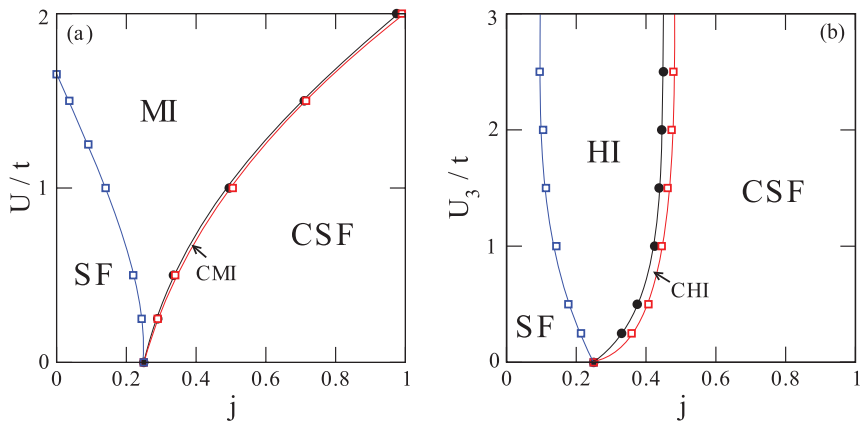

FIG. 2. (Color online) Phase diagram for unconstrained bosons as a function of the frustration parameter $j$ and (a) the on-site interaction $U$ (with $U_{3}=0$ ) and (b) the three-body repulsion $U_{3}$ (and $U=0$ ). In the figures, $\bigcirc$ indicates the boundary of the chiral phases characterized by long-range ordered chirality-chirality correlations $\left\langle\kappa_{i} \kappa_{j}\right\rangle ; \square$ indicates the boundary of the SF phases indicated by the critical Luttinger parameter $K=2$, which for the SF-MI and SF-HI Kosterlitz-Thouless (KT) transitions coincide well with the results from energy-level crossings. Note that narrow CMI and CHI phases may occur as well.

sound velocity, and $K$ is the Luttinger parameter. In weak coupling, $U m \ll 1$, hydrodynamic relations are expected to hold: $v_{s}(j) \sim \sqrt{\bar{n} U /\left(m \pi^{2}\right)}=v_{s}(0) \sqrt{1-4 j}$ and $K(j) \sim$ $\sqrt{\bar{n} \pi^{2} /(U m)}=K(0) \sqrt{1-4 j}$, clearly showing that $j$ enhances correlations. At $j=1 / 4, m$ diverges and the system enters a Mott insulator (MI) even for vanishingly small $U$ [Fig. 2(a)]. The SF-MI transition takes place, however, in the strong-coupling regime in which $v_{s}$ and $K$ must be determined numerically. We obtain $K$ from the single-particle correlations $G_{i j}=\left\langle b_{i} b_{j}^{\dagger}\right\rangle$, which in the SF decay as $\sim(-1)^{i-j}|i-j|^{-1 / 2 K}$. The value $K=2$ marks the boundary between $\mathrm{SF}\left(U<U_{c}\right.$, $K>2)$ and $\mathrm{MI}\left(U>U_{c}, K<2\right.$, and $\left.\mathcal{M}>0\right)$. The $\mathrm{MI}$ phase is characterized by a hidden parity order [19], $\mathcal{O}_{P}^{2}=$ $\lim _{|i-j| \rightarrow \infty}\left\langle(-1)^{\sum_{i<l<j} \delta n_{l}}\right\rangle \sim\langle\cos \sqrt{\pi} \phi\rangle^{2}$, which has been recently measured in site-resolved experiments [23].

The $j>1 / 4$ case is best understood from bosonization in the $j \gg 1$ regime. We may then introduce two pairs of bosonic fields $\left(\theta_{1}, \phi_{1}\right)$ and $\left(\theta_{2}, \phi_{2}\right)$, describing, respectively, the subchains of even and odd sites. The effective model is governed by the Hamiltonian density

$$
\begin{aligned}
\mathcal{H}= & \sum_{\alpha= \pm} \frac{v_{\alpha}}{2}\left[\frac{\left(\partial_{x} \phi_{\alpha}\right)^{2}}{K_{\alpha}}+K_{\alpha}\left(\partial_{x} \theta_{\alpha}\right)^{2}\right] \\
& +\lambda \partial_{x} \theta_{+} \sin \sqrt{2 \pi} \theta_{-}-2 \mathcal{M} \cos \sqrt{2 \pi} \phi_{+} \cos \sqrt{2 \pi} \phi_{-},
\end{aligned}
$$

where $\theta_{ \pm}=\left(\theta_{1} \pm \theta_{2}\right) / \sqrt{2}, \phi_{ \pm}=\left(\phi_{1} \pm \phi_{2}\right) / \sqrt{2}, v_{ \pm}, K_{ \pm}$, and $\mathcal{M}$ are phenomenological parameters [in the regimes displayed in Fig. 2(a)], and $\lambda \sim j^{-1}$. Note that the chirality is given by $\kappa_{i} \rightarrow \sin \sqrt{2 \pi} \theta_{-}(x)$. In weak coupling, $U m^{\prime} \ll 1$, with $m^{\prime}=\left[\partial^{2} \epsilon(k) / \partial k^{2}\right]_{k_{0}}^{-1}=4 j / t\left(16 j^{2}-1\right), v_{ \pm} \sim \sqrt{\bar{n} U /\left(m^{\prime} \pi^{2}\right)}$ and $K_{ \pm} \sim \sqrt{\bar{n} \pi^{2} /\left(U m^{\prime}\right)}$. In this case only the term $\partial_{x} \theta_{+} \sin \sqrt{2 \pi} \theta_{-}$is relevant, resulting in $\left\langle\sin \sqrt{2 \pi} \theta_{-}\right\rangle \neq 0$ [24]. Hence, a small $U$ is expected to favor a CSF for $j>1 / 4$, as our numerical results confirm [Fig. 2(a)]. The CSF phase is 
characterized by $G_{i j} \sim(-1)^{i-j} e^{-i \kappa(i-j)}|i-j|^{-1 / 4 K_{+}}$, where $\kappa \sim\left\langle\kappa_{i}\right\rangle$.

Moreover, depending on the values of $K_{ \pm}$, bosonization opens the possibility of two consecutive phase transitions with increasing $U$ starting from the CSF phase (Appendix A), which we have confirmed with our DMRG calculations [Fig. 2(a)] detailed in Appendix B. First a KT transition occurs from CSF to chiral Mott (CMI), a narrow Mott phase with finite chirality. Then an Ising transition is produced from CMI to nonchiral MI. At both KT transition lines in Fig. 2(a) (SF-MI and CSF-CMI), up to a logarithmic prefactor, $G_{i j} \sim$ $(-1)^{i-j} e^{-i \kappa(i-j)}|i-j|^{-1 / 4}$, where in CSF $\kappa \neq 0$.

\section{CONSTRAINED BOSONS}

As mentioned above, sufficiently large three-body losses may result in a three-body constraint $\left(b_{i}^{\dagger}\right)^{3}=0\left(U_{3}=\infty\right)[10]$. In that case, model (1) may be mapped to a large extent onto a frustrated spin-1 chain model [25], which presents the possibility of a gapped Haldane phase, characterized by a nonlocal string order. Hence, interestingly, constrained bosons in a zig-zag lattice may be expected to allow for the observation of the HI phase in the absence of polar interactions.

Indeed, a model with $U=0$ and finite $U_{3}$ shows that at the Lifshitz point, $j=1 / 4$, a HI phase is stabilized for arbitrarily weak $U_{3}$ [Fig. 2(b)]. The effective theory describing the $\mathrm{HI}$ is again the sine-Gordon model (2) with $K<2$. However, now $\mathcal{M}<0$, which selects a hidden string order $\mathcal{O}_{S}^{2} \equiv \lim _{|i-j| \rightarrow \infty}\left\langle\delta n_{i} \exp \left[i \pi \sum_{i<l<j} \delta n_{l}\right] \delta n_{j}\right\rangle \sim$ $\langle\sin \sqrt{\pi} \theta\rangle^{2}$ [19]. Resembling the case of Fig. 2(a), SF, HI, chiral-HI (CHI), and CSF phases occur [Fig. 2(b)]. These phases are expected for $U_{3}=\infty$ from known results in frustrated spin-1 chains [26-29]. Our DMRG simulations suggest that all these phases meet at $j=1 / 4$ for $U_{3} \rightarrow 0$.

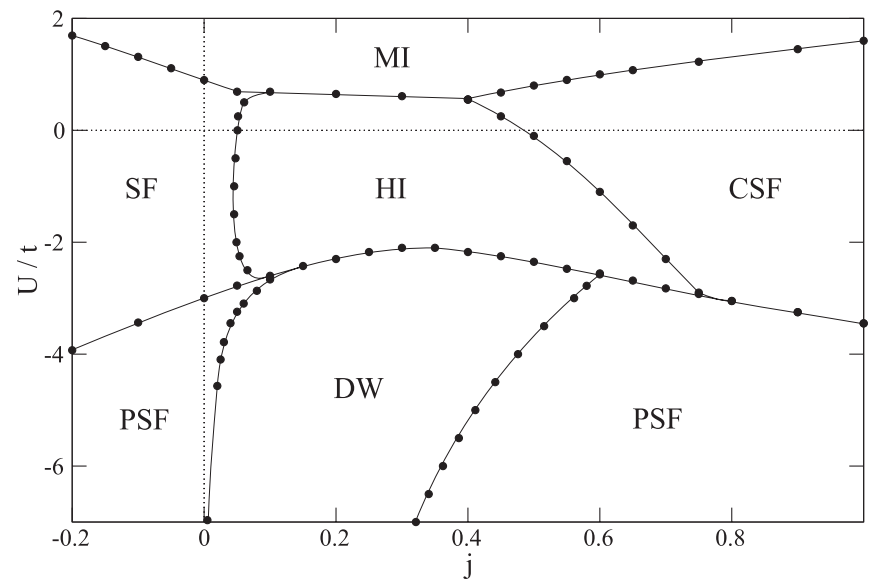

FIG. 3. Phase diagram for constrained bosons as a function of the on-site interaction $U$ and the frustration parameter $j$. Narrow CMI and CHI phases may occur along the phase-transition lines from CSF to MI and CSF to HI, respectively, but their extension would be negligible in the figure. For the precise location of the PSFDW and HI-MI transition we have additionally analyzed the energylevel crossings with, respectively, periodic- and twisted-boundary conditions. The KT transitions from SF to MI and HI have been determined by the extraction of the Luttinger parameter $K$ [32].
Figure 3 shows the phase diagram for constrained bosons $\left(U_{3}=\infty\right)$. Starting from the HI phase, increasing $U>0$ can induce a Gaussian HI-MI phase transition, characterized by a vanishing $\mathcal{M}=0$ in Eq. (2), resembling the phase transition between Haldane and large- $D$ phases induced by single-ion anisotropy in spin-1 chains [30]. The SF phase is separated from the MI and $\mathrm{HI}$ by KT transitions, whereas at the CSF boundary with the MI (HI) a CMI (CHI) occurs as mentioned above (these very narrow regions are not resolved in Fig. 3).

Interestingly, constrained bosons allow as well for the exploration of attractive two-body interactions, $U<0$, without collapse. The $U<0$ phases are also depicted in Fig. 3. For sufficiently large $|U|$, bosons tend to cluster in pairs and, as already discussed in Ref. [10], for $j=0$ an Ising transition between a SF and a pair superfluid (PSF) occurs [31], analogous to the $X Y 1$ to $X Y 2$ phase transition in spin- 1 chains induced by single-ion anisotropy [30] (this transition has been recently studied for two-dimensional lattices as well [12,14]). The PSF phase is characterized by an exponentially decaying $G_{i j}$ but algebraically decaying pair-correlation function $G_{i j}^{(2)}=$ $\left\langle\left(b_{i}^{\dagger}\right)^{2}\left(b_{j}\right)^{2}\right\rangle$. Indeed a PSF occurs for sufficiently large $|U|$, also for $j>1 / 4$ which is characterized in bosonization in Eq. (3) by a gapped antisymmetric sector, with pinned $\phi_{-}$, and a gapless symmetric sector [33]. Though one may anticipate an Ising phase transition between the CSF (with broken discrete parity symmetry) and the PSF (with restored symmetry), the behavior of $\mathcal{O}_{P}^{2}$ and $\kappa$ (not shown) hints to a weakly first-order nature.

Small $U<0$ disfavors singly occupied sites and thus enhances $\mathcal{O}_{S}^{2}$ and the bulk excitation gap of the HI phase (see Figs. 4 and 5). However, since large $U<0$ removes singly occupied sites completely, just like strong nearest-neighbor repulsion, it is expected that the HI phase eventually will transform for growing $|U|$ into a gapped density-wave (DW) phase via the Ising phase transition [16], and string order will evolve into DW order (Fig. 4 shows how $\mathcal{O}_{s}$ merges with $\mathcal{O}_{\text {DW }} \equiv \lim _{j \rightarrow \infty}(-1)^{j}\left\langle n_{i} n_{i+j}\right\rangle$ for $\left.U / t<-3\right)$. The DW phase is characterized by an exponential decay of both $G_{i j}$ and $G_{i j}^{(2)}$ though a finite $\mathcal{O}_{\text {DW }}$. Our DMRG results confirm this scenario (see Fig. 4), showing that a DW phase is located between the above-mentioned PSF regions (Fig. 3).

Interestingly the DW phase remains in between both PSF regions all the way to $U \rightarrow-\infty$. In that regime, we may project out singly occupied sites and introduce a pseudospin $1 / 2$, identifying $|0\rangle \rightarrow|\downarrow\rangle,|2\rangle \rightarrow|\uparrow\rangle$, and defining the spin

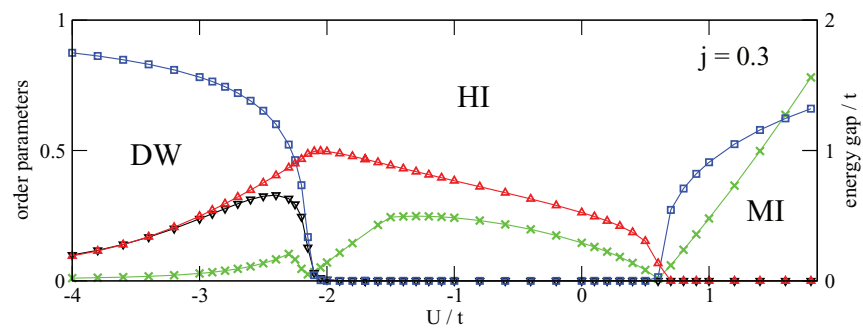

FIG. 4. (Color online) Order parameters $\mathcal{O}_{P}^{2}(\square), \mathcal{O}_{S}^{2}(\triangle)$, $\mathcal{O}_{\text {DW }}(\nabla)$, and energy gap $(\times)$ as functions of $U$ for constrained bosons on the $j=0.3$ line $(N=160)$. The parity, as defined in the Mott state, must get an additional oscillating factor in the DW phase $\mathcal{O}_{P}^{2} \rightarrow(-1)^{i-j} \mathcal{O}_{P}^{2}$ 


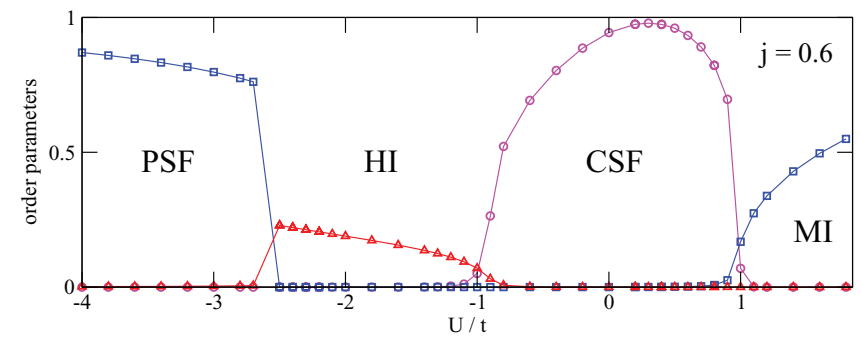

FIG. 5. (Color online) Order parameters $\mathcal{O}_{P}^{2}(\square), \mathcal{O}_{S}^{2}(\triangle)$, and $\kappa^{2}$ ( $\bigcirc$ ) as functions of $U$ for constrained bosons on the $j=0.6$ line $(N=160)$. Both $\mathcal{O}_{P}^{2}$ and $\mathcal{O}_{S}^{2}$ show jumps at the $\mathrm{HI}$ to PSF transition at $U \simeq-2.5$. In the PSF, similar to the DW phase, $\mathcal{O}_{P}^{2}$ is defined with an additional oscillating factor.

operators $\tau_{i}^{-} \rightarrow(-1)^{i} b_{i}^{2} / \sqrt{2}, 2 \tau_{i}^{z} \rightarrow b_{i}^{\dagger} b_{i}-1$. The effective model to leading order in $1 /|U|$ is a spin- $1 / 2$ chain:

$$
H_{\frac{1}{2}}=J \sum_{i}\left[\boldsymbol{\tau}_{i} \boldsymbol{\tau}_{i+1}+j^{2}\left(\tau_{i}^{z} \tau_{i+2}^{z}-\tau_{i}^{x} \tau_{i+2}^{x}-\tau_{i}^{y} \tau_{i+2}^{y}\right)\right],
$$

where $J=t^{2} /|U|$. For $j=0$, this is a $\mathrm{SU}(2)$ symmetric chain, whereas the $j^{2} J$ terms break the symmetry down to $\mathrm{U}(1)$, moving the effective theory obtained after bosonization of $H_{\frac{1}{2}}$ towards the irrelevant direction (in the renormalization-group sense). As a result of this, a gapless $X Y$ phase of the spin-1/2 chain is expected, i.e., a PSF phase. Higher-order terms in $1 /|U|$ (not shown explicitly) break, even for $j=0$, the $\mathrm{SU}(2)$ symmetry to $\mathrm{U}(1)$ in the irrelevant direction. However, interestingly, the ring exchange along the elementary triangle of the zig-zag chain, with amplitude $j t^{3} / U^{2}$, forces the effective theory towards the relevant direction, leading to a gapped Néel phase of the spin-1/2 chain, i.e., the DW phase. The competition between exchange along the lattice bonds and ring-exchange leads hence to two consecutive KT phase transitions induced by $j$, for $j \ll 1$ first from PSF to DW, followed by DW back to PSF. The width of the DW phase is $\sim t /|U|$, and it extends all the way into the $U \rightarrow-\infty$ limit.

Finally, our DMRG simulations show a narrow region where a direct, apparently first-order, HI-PSF transition occurs (for details see Appendix B), characterized by discontinuous jumps of $\mathcal{O}_{S}^{2}$ and $\mathcal{O}_{P}^{2}$ (Fig. 5). This first-order nature is explained because, on one hand, increasing $|U|$ within the HI phase increases $\mathcal{O}_{S}^{2}$ due to the suppression of singly occupied sites and, on the other hand, for $0.6 \lesssim j \lesssim 0.75$ (Fig. 3), a growing $|U|$ destroys the insulating state in favor of a PSF phase, where string order cannot exist. On the contrary, $\mathcal{O}_{S}^{2}$ diminishes for decreasing $|U|$ when approaching the HI-CSF boundary (Fig. 5).

\section{CONCLUSIONS}

In conclusion, the interplay between geometrical frustration and interactions leads to rich physics for ultracold bosons in zig-zag optical lattices. Unconstrained bosons may present chiral superfluidity and Mott insulator for vanishingly small interactions. Constrained bosons may allow for the observation of a Haldane insulator without the necessity of polar interac- tions, as well as pair-superfluid and density-wave phases at attractive interactions.

Although our paper deals only with zero-temperature physics, we would like to comment briefly on the case of finite temperature. Experiments on cold gases deal with finite-size systems where in order to observe the corresponding ground-state orders the temperature should be lowered below the characteristic energy scale, which is set by the magnitudes of the zero-temperature gaps $\Delta E$ in the excitation spectrum. For the PSF phase, the characteristic energy scale is set by the single-particle excitation gap that scales linearly with $|U|$ for negative $U$ in strong coupling, $|U| \gg t$. As depicted in Fig. 4, for $j=0.3$, the excitation gap can reach in the HI phase $\Delta E \sim t / 2$ for $U \simeq-1.5 t$ and in the DW phase $\Delta E \simeq t / 4$ for $U \simeq-2.2 t$.

All the predicted phases may be detected using state-of-theart techniques. The SF and CSF phases may be distinguished by means of time-of-flight (TOF) techniques, in a similar way as recently done for condensates in triangular optical lattices [8]. The DW and PSF phases are characterized by double or zero occupancy, which could be detected using parity measurements such as those introduced in Refs. [34,35] and could be discerned from each other by the absence or presence of interference fringes in TOF [36]. Finally, the string-order of the HI phase may be studied using similar site-resolved measurements such as those recently reported for the measurement of nonlocal parity order in Mott insulators [23].

\section{ACKNOWLEDGMENT}

This work has been supported by the cluster of excellence Center for Quantum Engineering and Space-Time Research (QUEST). T.V. acknowledges SCOPES Grant IZ73Z0-128058.

\section{APPENDIX A: BOSONIZATION ANALYSIS OF MI-CMI-CSF TRANSITION}

In this Appendix we provide additional details on our bosonization analysis. We take the limit $j \gg 1$, which allows us to consider subchains formed by the even and odd sites and to introduce the symmetric and antisymmetric fields $\theta_{ \pm}$and $\phi_{ \pm}$. The interaction between these fields is given by the last two terms of Eq. (3) of the main text:

$$
\mathcal{H}_{\text {int }}=\lambda \partial_{x} \theta_{+} \sin \sqrt{2 \pi} \theta_{-}-2 \mathcal{M} \cos \sqrt{2 \pi} \phi_{+} \cos \sqrt{2 \pi} \phi_{-},
$$

where the first term supports chirality and the second one favors a MI phase.

Starting from $U \gg t^{\prime}$, deep in the Mott phase of each subchain, then $\left\langle\cos \sqrt{2 \pi} \phi_{ \pm}\right\rangle \neq 0$, and thus the $\phi_{ \pm}$fields are pinned in the Mott phase. In this case, to second order in $\lambda$, one can integrate out in the partition function $\partial_{x} \theta_{+}$from the first term of Eq. (A1), obtaining the following contribution in the antisymmetric sector:

$$
-\frac{\lambda^{2}}{2} \int d^{2} x d^{2} y\left\langle\partial_{x} \theta_{+} \partial_{y} \theta_{+}\right\rangle \sin \sqrt{2 \pi} \theta_{-}(x) \sin \sqrt{2 \pi} \theta_{-}(y),
$$


where the average is performed in the ground state of the MI phase, where $\left\langle\partial_{x} \theta_{+} \partial_{y} \theta_{+}\right\rangle$is short ranged. Hence the leading contribution in the antisymmetric sector, after carrying out operator product expansion, is a term $\sim \lambda^{2} \cos \sqrt{8 \pi} \theta_{-}$. Note that an $\sim-\lambda^{2}\left(\partial_{x} \theta_{-}\right)^{2}$ contribution decreasing the value of $K_{-}$is obtained as well in the antisymmetric sector. The competition between $\cos \sqrt{8 \pi} \theta_{-}$and $\cos \sqrt{2 \pi} \phi_{-}$[obtained using mean-field decoupling of the last term of Eq. (A1) in the MI phase] is resolved with an Ising phase transition in the antisymmetric sector with increasing $\lambda / U$, leading to the pinning of $\theta_{-}$in the new ground state $\left\langle\sqrt{8 \pi} \theta_{-}\right\rangle=\pi$, so that $\left\langle\sin \sqrt{2 \pi} \theta_{-}\right\rangle \neq 0$, driving the symmetric sector into a state with finite topological current, $\left\langle\partial_{x} \theta_{+}\right\rangle \neq 0$.

The simplest scenario to establish the Ising phase transition is for $K_{-}=2$. In that case, performing a mean-field decoupling of the second term in Eq. (A1), the antisymmetric sector is governed by the Hamiltonian density,

$$
\begin{aligned}
\mathcal{H}_{-}= & \frac{v_{-}}{2}\left[\left(\partial_{x} \tilde{\phi}_{-}\right)^{2}+\left(\partial_{x} \tilde{\theta}_{-}\right)^{2}\right] \\
& -\tilde{M} \cos \sqrt{4 \pi} \tilde{\phi}_{-}+\tilde{\lambda} \cos \sqrt{4 \pi} \tilde{\theta}_{-},
\end{aligned}
$$

where $\tilde{\theta}_{-}=\sqrt{K_{-}} \theta_{-}, \quad \tilde{\phi}_{-}=\phi_{-} / \sqrt{K_{-}}, \quad \tilde{M}=2 \mathcal{M}\langle\cos \sqrt{2 \pi}$ $\left.\phi_{+}\right\rangle$, and $\tilde{\lambda} \sim \lambda^{2}$. The antisymmetric sector can be hence described by two free massive Majorana fermions, with masses $\tilde{\lambda} \pm \tilde{M}$. At the Ising phase transition, $\tilde{\lambda}= \pm \tilde{M}$, and the mass of one of the Majorana fermions vanishes [37].

However, the Mottness of the ground state after the chirality gets ordered over long range, $\left\langle\sqrt{2 \pi} \theta_{-}\right\rangle \neq 0$, does not necessarily disappear immediately due to the possibility of a relevant contribution in the symmetric sector, $\cos \sqrt{8 \pi} \phi_{+}$, for $K_{+}<1$ which stems after integrating out the $\cos \sqrt{2 \pi} \phi_{-}$in the last term of Eq. (A1) in the state with pinned $\theta_{-}$. Note that in the CMI state $\left\langle\theta_{-}\right\rangle \neq 0,\left\langle\partial_{x} \theta_{+}\right\rangle \neq 0$, and also $\left\langle\phi_{+}\right\rangle \neq 0$. Further decreasing $U$, at $K_{+}=1$, the CMI phase $\left(K_{+}<1\right)$ disappears at a KT phase transition in favor of the CSF phase $\left(K_{+}>1\right)$.

Thus, our bosonization analysis, for $j \gg 1$, suggests the possibility of two consecutive phase transitions with increasing $U$ starting from the CSF phase: first a KT transition from CSF to CMI, followed by an Ising transition from CMI to MI. Note that if an intermediate CMI phase between CSF and MI is absent, the direct transition between CSF and MI cannot be of Ising nature, since CSF is a gapless phase and MI is gapped. In the following section, we provide numerical proof of the existence of the CMI phase, showing that starting from the MI phase, the system experiences an Ising transition involving the growth of chirality.

\section{APPENDIX B: NUMERICAL ANALYSIS}

Here we provide details of our numerical calculations, and in particular on how phase boundaries were determined and how error bars were estimated.

We investigate the phase diagrams by means of numerical simulations based on exact diagonalization and density-matrix renormalization group (DMRG) with up to 300 lattice sites. Typically, for DMRG simulations we keep about $\chi \approx 500$ matrix states. The results have also been confirmed by an infinite-system-size algorithm (iDMRG) [38] with up to

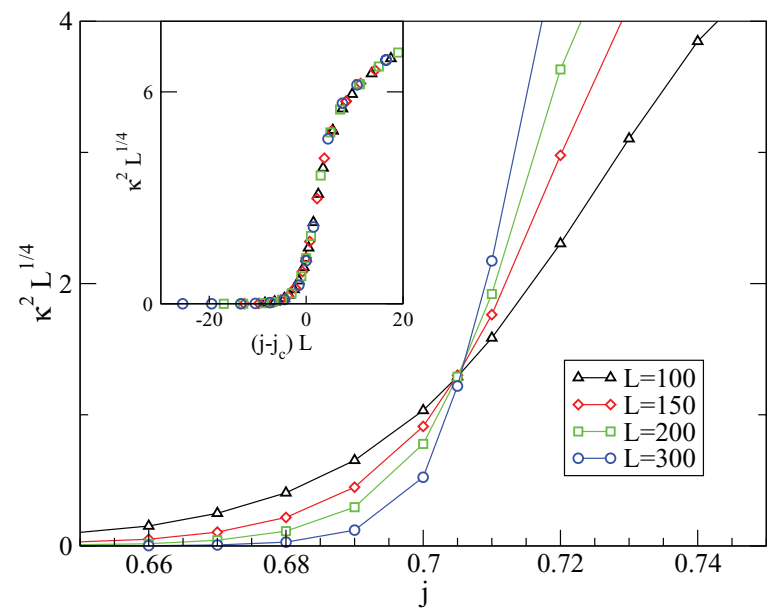

FIG. 6. (Color online) Chirality scaling $\kappa^{2} L^{1 / 4}$ close to MI-CMI transition for $U=3$. The inset shows the collapse of all finite-systemsize data to a single curve. The results indicate a MI-CMI Ising transition at $j=0.705 \pm 0.001$.

$\chi \approx 300$ states. For our simulations of the unconstrained Bose-Hubbard model we kept $n_{\max }=4$ bosons per site for $U \geqslant 1$ and $n_{\max }=6$ for $U=0.5$, which has been shown to be sufficient by comparing to simulations with higher $n_{\max }$. For open-boundary conditions special care may be needed to take care of degenerate edge states in the Haldane phase, i.e., by polarizing edges [16].

First we discuss phase transitions involving chiral order parameter $\left\langle\kappa_{i}\right\rangle$, where $\kappa_{i}$ is defined in Sec. III. Since the chiral order is spontaneous, we study numerically the chiralitychirality correlation function $\left\langle\kappa_{i} \kappa_{i+n}\right\rangle$ [28], which at large distances saturates to $\kappa^{2}$. We study how the chirality vanishes with changing $j$ (for the MI-CMI and HI-CHI transitions; for the CSF-PSF transition we study instead the behavior of the parity order). The scaling of chirality $\kappa^{2} L^{1 / 4}$ close to MI-CMI and HI-CHI transitions unambiguously confirms that the corresponding phase transition is of Ising type, showing the correct scaling behavior. The critical value of $j$ for the corresponding Ising transition is located by extracting the intersection of curves for different system sizes $L$ (the width of the intersecting point provides the uncertainty of the procedure). Figure 6 illustrates our numerical results in the vicinity of the MI-CMI transition. The collapse of the data for different system sizes on a single curve (inset) confirms the Ising nature of the underlying phase transition. Similarly, we confirm numerically the Ising nature of the HI-CHI transition (not shown).

The standard numerical procedure to locate the KT transition from superfluid to gapped phase is based on the Luttinger liquid parameter, which we extract from the singleparticle correlation function $G_{i j}$. When the single-particle correlations show incommensurate oscillations, we fit it to $G_{i j} \propto G(r)^{1 / 2 K} \cos (\omega x+\phi)$ with $G(r)$ including conformal corrections [39]. To get a lower bound for the transition point one can apply a power-law fit to shorter distances after dividing out incommensurate oscillations. The KT CMI-CSF transition (as well as the CHI-CSF transition) can be located in this way providing a strong hint of the existence of a finite CMI 

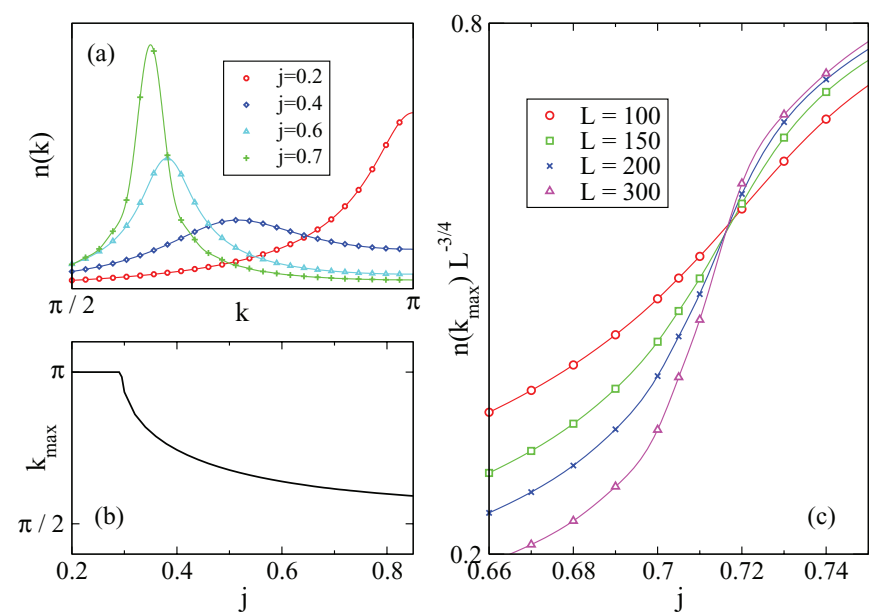

FIG. 7. (Color online) (a) Momentum distribution $n(k)$ for unconstrained bosons, for $U=3$ and $L=100$ (the position of the maximum $k_{\max }$ is practically independent of $L$ ). (b) Value of $k_{\max }$ as a function of $j$. At $j \approx 0.3$ the quasimomentum peak departs from $\pi$ and $n(k)$ acquires two inequivalent maxima at $\pm k_{\max }$. (c) Scaling of $n\left(k_{\max }\right) L^{-3 / 4}$ for different $L$. The crossing of the curves indicates the CMI-CSF transition at $j=0.717 \pm 0.001$. Extracting the Luttinger liquid parameter as indicated in the text gives a similar estimate of $j=0.72 \pm 0.01$ though with an order of magnitude larger error bar.

(CHI) region between the $\mathrm{MI}(\mathrm{HI})$ and CSF phases. However, a much more accurate estimate of the KT transition point is provided by the analysis of the quasimomentum distribution $n(k)$ [40]. Since $G_{i j} \sim e^{-i Q(i-j)}|i-j|^{-\alpha}$ with $\alpha=1 / 4$ at the KT transition (up to logarithmic correction), $n(k)$ has a maximum at $Q$ and its height depends on the system size as $n(Q) \approx \frac{1}{L} \sum_{i, j} e^{i Q(i-j)} G_{i j} \sim L^{1-\alpha}$. This behavior is illustrated in Fig. 7, which shows a clear intersection of the $n\left(k_{\max }\right) L^{\alpha-1}$ curves for different system sizes at a single point (the width of this point provides the error bar for the position of the KT transition). Hence for the case depicted in Figs. 6 and 7, a narrow but clearly determined CMI phase may be found between $j=0.705 \pm 0.001$ and $j=0.717 \pm 0.001$.

In addition to chirality we have studied other order parameters, including parity, string, and DW orders (all defined in the main text). Applying finite-size-scaling analysis [41] allows an accurate location of the corresponding phase-transition lines depicted in Fig. 3 of the main text.

In Fig. 8 we depict the scaling of the parity order in the vicinity of PSF-SF and PSF-CSF transitions using the iDMRG algorithm. The correlation length for the quantum Ising model scales like $\xi=\chi^{2}$, where $\chi$ denotes the matrix dimension [42]. This result is in very good agreement with our results for the PSF-SF transition (Fig. 8, left). Indeed, using Ising critical exponents the parity order parameter behaves as

$$
\mathcal{O}_{P}^{2}=\xi^{-1 / 4} f\left(\left(U-U_{c}\right) \xi\right) \text {. }
$$

On the contrary, for the CSF-PSF transition the behavior is fundamentally different, and we instead observe a jump in the parity order (Fig. 8, right), indicating a first-order character of the transition. We observe as well a similar jump in the parity order across the PSF-HI transition. In addition string
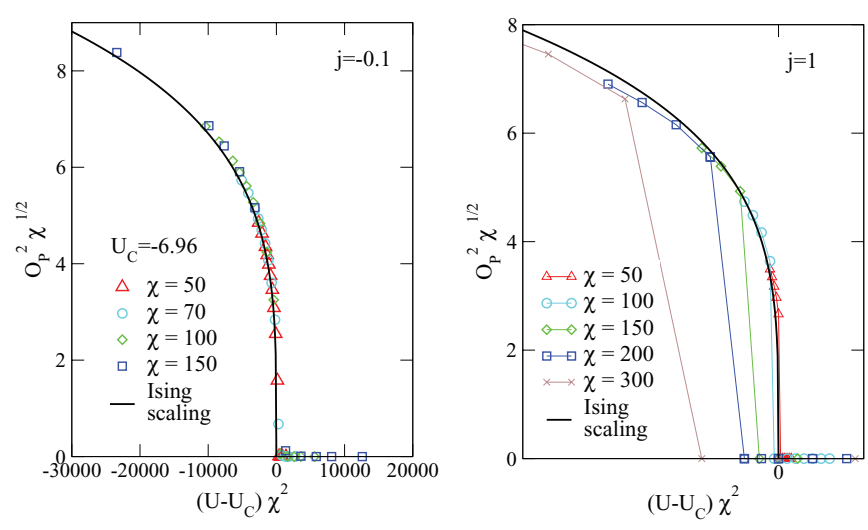

FIG. 8. (Color online) (left) Scaling with the matrix dimension $\chi$ of the parity order parameter for the PSF-SF transition. Note that the scaling accurately follows that expected for an Ising transition. (right) Same results for the PSF-CSF transition show clear deviations from the expected Ising scaling, exhibiting an abrupt jump in the parity order.

order shows an abrupt jump at the same transition as depicted in Fig. 9.

Besides monitoring order parameters we use a finite-size level-crossing analysis (level spectroscopy) [43] to determine the location of different phase transitions. We use DMRG for longer chains, which compares well with our exact diagonalization results available only up to 16 sites. The SF-PSF transition can be extrapolated very precisely from level crossing between the one-particle and two-particle excitation gaps. We determine in this way the SF-PSF boundary, which compares well with the boundary obtained from monitoring the parity order. The transitions from DW to PSF phases can also be determined very accurately by level spectroscopy. Finite-size extrapolation following $1 / N^{2}$ law confirms the KT nature of those transitions. Finally, for twisted boundary conditions the Gaussian transition line between HI and MI can also be located by ground-state level crossing [44].

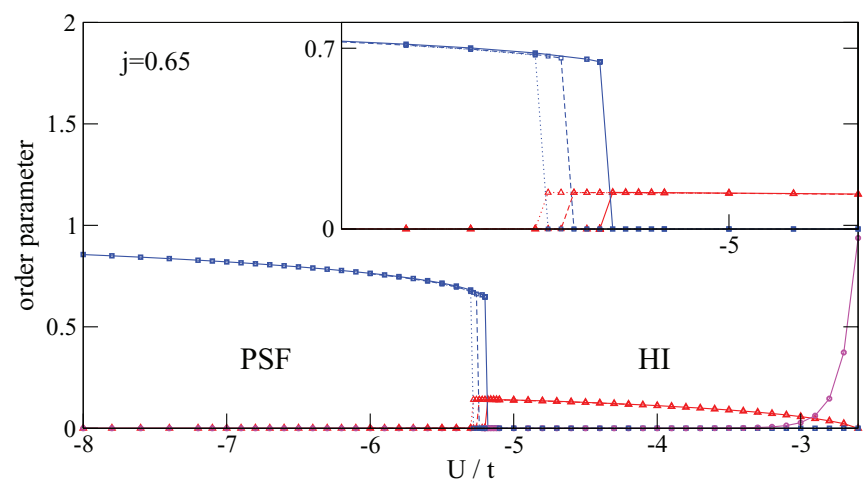

FIG. 9. (Color online) Parity order $\mathcal{O}_{P}^{2}(\square)$, string order $\mathcal{O}_{S}^{2}(\triangle)$, and chirality $\kappa^{2}(\bigcirc)$ for constrained bosons $(j=0.65)$ close to the HI-PSF transition of iDMRG calculations for different matrix dimensions: $\chi=100$ (straight line), $\chi=200$ (dashed line), $\chi=300$ (dotted line). The transition point shifts slightly with increasing $\chi$. The inset shows a zoom in the HI-PSF transition region. 
[1] M. Lewenstein et al., Adv. Phys. 56, 243 (2007).

[2] V. I. Yukalov, Laser Phys. 19, 1 (2009).

[3] C. Becker et al., New J. Phys. 12, 065025 (2010).

[4] G.-B. Jo, J. Guzman, C. K. Thomas, P. Hosur, A. Vishwanath, and D. M. Stamper-Kurn, Phys. Rev. Lett. 108, 045305 (2012).

[5] S. D. Huber and E. Altman, Phys. Rev. B 82, 184502 (2010).

[6] A. Eckardt, C. Weiss, and M. Holthaus, Phys. Rev. Lett. 95, 260404 (2005).

[7] A. Zenesini, H. Lignier, D. Ciampini, O. Morsch, and E. Arimondo, Phys. Rev. Lett. 102, 100403 (2009).

[8] J. Struck et al., Science 333, 996 (2011).

[9] C. Chin et al., Rev. Mod. Phys. 82, 1225 (2010).

[10] A. J. Daley, J. M. Taylor, S. Diehl, M. Baranov, and P. Zoller, Phys. Rev. Lett. 102, 040402 (2009).

[11] I. Titvinidze et al., New J. Phys. 13, 035013 (2011).

[12] L. Bonnes and S. Wessel, Phys. Rev. Lett. 106, 185302 (2011).

[13] M. Dalmonte, M. DiDio, L. Barbiero, and F. Ortolani, Phys. Rev. B 83, 155110 (2011).

[14] Y.-C. Chen, K.-K. Ng, and M.-F. Yang, Phys. Rev. B 84, 092503 (2011).

[15] M. J. Mark, E. Haller, K. Lauber, J. G. Danzl, A. Janisch, H. P. Buchler, A. J. Daley, and H. C. Nagerl, Phys. Rev. Lett. 108, 215302 (2012).

[16] E. G. Dalla Torre, E. Berg, and E. Altman, Phys. Rev. Lett. 97, 260401 (2006).

[17] T. Lahaye et al., Rep. Prog. Phys. 72, 126401 (2009).

[18] F. D. M. Haldane, Phys. Lett. A 93, 464 (1983); Phys. Rev. Lett. 50, 1153 (1983).

[19] E. Berg, E. G. Dalla Torre, T. Giamarchi, and E. Altman, Phys. Rev. B 77, 245119 (2008).

[20] M. den Nijs and K. Rommelse, Phys. Rev. B 40, 4709 (1989).

[21] S. R. White, Phys. Rev. Lett. 69, 2863 (1992); Phys. Rev. B 48, 10345 (1993).

[22] T. Giamarchi, Quantum Physics in One Dimension (Oxford University Press, Oxford, UK, 2003).

[23] M. Endres et al., Science 334, 200 (2011).

[24] A. A. Nersesyan, A. O. Gogolin, and F. H. L. Essler, Phys. Rev. Lett. 81, 910 (1998).

[25] One may map the boson problem by means of the HolsteinPrimakoff transformation $S_{i}^{z}=1-n_{i}, S_{i}^{+}=\sqrt{2-n} b_{i}$ to a frustrated spin-1 $X Y$ chain (up to particle-hole symmetry breaking terms that are irrelevant [19]), $H_{S}=t \sum_{i}\left[\left(\vec{S}_{i}\right.\right.$. $\left.\left.\vec{S}_{i+1}\right)_{X Y}+j\left(\vec{S}_{i} \cdot \vec{S}_{i+2}\right)_{X Y}+D\left(S_{i}^{z}\right)^{2}\right]$, where $\left(\vec{S}_{i} \cdot \vec{S}_{j}\right)_{X Y} \equiv S_{i}^{x}$. $S_{j}^{x}+S_{i}^{y} \cdot S_{j}^{y}$, and $D=U /(4 t)$ and $j$ characterize, respectively, the single-ion anisotropy and the next-nearest neighbor frustrating exchange.

[26] A. K. Kolezhuk, Prog. Theor. Phys. Suppl. 145, 29 (2002); Phys. Rev. B 62, R6057 (2000).

[27] P. Lecheminant, T. Jolicoeur, and P. Azaria, Phys. Rev. B 63, 174426 (2001).

[28] T. Hikihara et al., J. Phys. Soc. Jpn. 69, 259 (2000).

[29] T. Hikihara, J. Phys. Soc. Jpn. 71, 319 (2002).

[30] H. J. Schulz, Phys. Rev. B 34, 6372 (1986).

[31] Numerically we have unambiguously confirmed the Ising nature of the SF-PSF transition by observing the correct scaling of $\mathcal{O}_{P}^{2}$ close to the transition point.

[32] It is notoriously hard to determine precisely the KT phasetransition lines by this method alone. The precise location of the phase-transition lines, and the determination of the nature of the multicritical points close to $j=0$ are not pursuit in the current work.

[33] T. Vekua, A. Honecker, H. J. Mikeska, and F. Heidrich-Meisner, Phys. Rev. B 76, 174420 (2007).

[34] W. S. Bakr et al., Science 329, 547 (2010).

[35] J. F. Sherson et al., Nature (London) 467, 68 (2010).

[36] M. Greiner et al., Nature (London) 415, 39 (2002).

[37] A. O. Gogolin, A. A. Nersesyan, and A. M. Tsvelik, Bosonization and Strongly Correlated Systems (Cambridge University Press, Cambridge, UK, 1998).

[38] U. Schollwöck, Rev. Mod. Phys. 77, 259 (2005).

[39] M. A. Cazalilla, J. Phys. B 37, (2004).

[40] A. Dhar, M. Maji, T. Mishra, R. V. Pai, S. Mukerjee, and A. Paramekanti, Phys. Rev. A 85, 041602 (2012).

[41] H. Ueda, H. Nakano, and K. Kusakabe, Phys. Rev. B 78, 224402 (2008).

[42] L. Tagliacozzo, T. R. de Oliveira, S. Iblisdir, and J. I. Latorre, Phys. Rev. B 78, 024410 (2008).

[43] K. Nomura, J. Phys. A 28, 5451 (1995); A. Kitazawa, ibid. 30, L285 (1997); T. Murashima, K. Hijii, K. Nomura, and T. Tonegawa, J. Phys. Soc. Jpn. 74, 1544 (2005).

[44] W. Chen, K. Hida, and B. C. T. Sanctuary, Phys. Rev. B 67, 104401 (2003). 